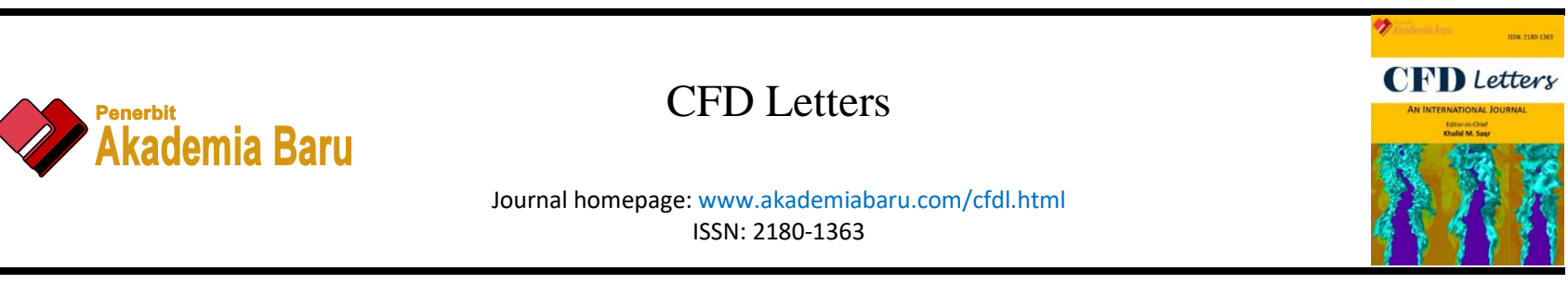

\title{
Effect of Cylinder Gap Ratio on The Wake of a Circular Cylinder Enclosed by Various Perforated Shrouds
}

\author{
Nurul Azihan Ramli ${ }^{1}$, Azlin Mohd Azmi ${ }^{1,2,}{ }^{*}$, Ahmad Hussein Abdul Hamid ${ }^{1}$, Zainal Abidin Kamarul \\ Baharin $^{1}$, Tongming Zhou ${ }^{3}$ \\ 1 School of Mechanical Engineering, College of Engineering, Universiti Teknologi Malaysia, 40450 Shah Alam, Selangor, Malaysia \\ Solar Research Institute (SRI), Universiti Teknologi MARA, 40450 Shah Alam, Selangor, Malaysia \\ 3 Department of Civil, Environmental and Mining Engineering, The University of Western Australia, 35 Stirling Highway, Crawley, WA 6009, \\ Australia
}

\section{ARTICLE INFO}

Article history:

Received 5 May 2020

Received in revised form 26 April 2021

Accepted 27 April 2021

Available online 27 April 2021

Keywords:

Perforated shroud; vortex shedding; passive control

\section{ABSTRACT}

\begin{abstract}
Flow over bluff bodies produces vortex shedding in their wake regions, leading to structural failure from the flow-induced forces. In this study, a passive flow control method was explored to suppress the vortex shedding from a circular cylinder that causes many problems in engineering applications. Perforated shrouds were used to control the vortex shedding of a circular cylinder at Reynolds number, $\operatorname{Re}=200$. The shrouds were of non-uniform and uniform holes with $67 \%$ porosity. The spacing gap ratio between the shroud and the cylinder was set at 1.2, 1.5, 2, and 2.2. The analysis was conducted using ANSYS Fluent using a viscous laminar model. The outcomes of the simulation of the base case were validated with existing studies. The drag coefficient, $\mathrm{Cd}$, lift coefficient, $\mathrm{Cl}$ and the Strouhal number, St, as well as vorticity contours, velocity contours, and pressure contours were examined. Vortex shedding behind the shrouded cylinders was observed to be suppressed and delayed farther downstream with increasing gap ratio. The effect was significant for spacing ratio greater than 2.0 . The effect of hole types: uniform and non-uniform holes, was also effective at these spacing ratios for the chosen Reynolds number of 200. Specifically, a spacing ratio of 1.2 enhanced further the vortex intensity and should be avoided.
\end{abstract}

\section{Introduction}

Flow over bluff bodies has many applications in engineering. This kind of flow will produce vortex shedding in the wake region. According to Dehkordi et al., [1], these bodies, subjected to air or water flow may experience flow-induced forces that can lead to failure over time. Vortex shedding occurs when the flow reaches a critical Reynolds number. The Reynolds number can be classified into different regimes such as subcritical, critical, supercritical and transcritical. The behaviour of vortex shedding is dependent upon the Reynolds number regime.

When vortex shedding degenerates to lock-in condition, it will become a complex physical phenomenon as large vibrations may occur at moderate and frequent fluid velocities. Bodies may

\footnotetext{
* Corresponding author.

E-mail address: azlinazmi@uitm.edu.my (Azlin Mohd Azmi)
} 
undergo many stress cycles that lead to damage and may influence structural failure without exceeding the ultimate limit stress, according to Giosan and Eng [2]. It is crucial to design a structure that can modify this flow considering the potential vortex shedding fatigue-induced damage called the Vortex-Induced Vibration (VIV).

Galbraith [3] observed that this VIV phenomenon could cause accelerated fatigue damage to structures, such as a circular cylinder. The VIV of cylindrical structures occurs in many engineering applications such as cooling towers, transmission cables, and offshore components. Starting at a low Reynolds number of about 50, bluff bodies placed within an external flow form an unstable wake that results in developing a regular vortices pattern Karman street, as observed by Triantafyllou et al., [4].

The control of the wake region becomes a challenging factor in many fields of engineering. The wake and vortex shedding can be controlled either by passive or active method. Cottet et al., [5] used an active control approach, imparting energy on the flow-through actuators on the surfaces of the obstacles. This method involves cost to implement as it requires an additional device to control the vortex. Meanwhile, passive control method focuses on the adjustment to the geometry of the body itself. The passive flow control method has attracted great attention by researchers as a method to suppress the vortex in recent decades. According to Every et al., [6], flow can be controlled by altering the structural profile to affect the separation of the boundary layer to prevent vortex shedding along the structure's length or change the formation of the wake vortices and their interactions. Several passive methods can be used to suppress vortex shedding, such as Helical Strakes, Control Plates, and Perforated Cylinder which usually involves geometrical modification.

Boorsma et al., [7] studied the effect of perforated fairings to improve the noise control of landing gears. The porosity, $\beta$, and location of perforations on the fairings were applied to simplified landing gear models and were shown to reduce the low-frequency noise. According to Ikeda and Takaishi [8], stable wake shear layers of the perforated cylinder and suppression of Aeolian tone were achieved due to the jets emitted from the holes at regular intervals. Kleissl and Georgakis [9] performed an experimental study to illustrate the effect of modifying bridge cable shape and surface on suppression of the VIV. They discovered that a shrouded cylinder reduced the vortex-induced oscillating lift forces. Experiments conducted by Osrin et al., [10] on the effect of shroud hole uniformity on a circular cylinder wake showed that, although all shrouded cylinder models reduced drag significantly in comparison to that of the bare cylinder case, the non-uniform hole shrouds were considerably effective than those of uniform hole shrouds. Misman et al., [11] discovered that simply changing the slot profile can prevent vortex shedding along the structure's length and wake vortices' formation.

Yao et al., [12] analyzed the Reynolds number's effect on smooth circular cylinders' drag coefficient. In addition, Sudalaimuthu and Liu [13] also compared a smooth circular cylinder and perforated circular cylinder for reduction in flow-induced vibrations (FIV) at the Reynolds number range $0.5 \times 10^{5}$ to $2.0 \times 10^{5}$. The perforated circular cylinder proved to reduce drag and lift coefficients more than the smooth cylinder, which shows a beneficial reduction in FIV. An experiment on the flow past a perforated conic shroud at Reynold number of 100 performed by Lin et al., [14] showed that the attack of angle increment and hole diameter were barely affecting the drag of the perforated shroud. The lift, however, decreased with increasing hole diameter. Azmi and Zhou [15] studied the effect of screen geometry on vortex formation behind a circular cylinder using flow visualizations. The study proposed a new dimensionless parameter, combining the effect of both screen wire and circular cylinder geometry in regulating the vortex shedding in the wake of a circular cylinder. Remarkable suppression was also obtained with higher gap ratio at Reynolds number of 1000 . Steiros 
et al., [16] performed a study on different porous shapes such as plates and cylinders. Unlike a flat plate, the cylinder's perforation can lower its drag as porosity becomes bigger when exposed to flow.

In this paper, a circular cylinder enclosed in a perforated circular shroud of different hole types (uniform and non-uniform holes) is studied to examine the effect of the gap ratio and shroud's perforation geometry on the circular cylinder wake at low Reynolds number. This serves as a preliminary study and contribute to the body of knowledge on shroud's perforation and gap ratio effect in suppressing vortex behind a cylinder, which is scarce in the literature.

\section{Methodology}

The study starts with validating simulation of flow over a circular cylinder with the previous experimental study by other researchers as shown in Table 1. The purpose of the validation is to ensure that the simulation setup and model used are suitable and produce reliable results. The circular cylinder's simulation result is compared with the existing study and utilized the same setup to simulate other cases. The percentage of differences that had been accepted for validation result is between 1 per cent and 10 per cent. Three stages are involved in the simulation: Pre-Processing Stage, Computational Stage, and Post-processing Stage.

Table 1

Result of simulation

\begin{tabular}{lll}
\hline & Drag Coefficient, $C_{d}$ & Strouhal Number, St \\
\hline Circular cylinder & & \\
Meneghini et al., [17] & 1.30 & 0.196 \\
Ding et al., [18] & 1.34 & 0.196 \\
Present & 1.299 & 0.190 \\
67\% porosity perforated shrouded cylinder with gap spacing ratio 1.2 & \\
Uniform & 1.461 & 0.165 \\
Non-uniform & 1.472 & 0.165 \\
67\% porosity perforated shrouded cylinder with gap spacing ratio 1.5 & \\
Uniform & 1.008 & 0.135 \\
Non-uniform & 0.978 & 0.135 \\
67\% porosity perforated shrouded cylinder with gap spacing ratio 2 & \\
Uniform & 0.665 & 0.089 \\
Non-uniform & 0.627 & 0.089 \\
67\% porosity perforated shrouded cylinder with gap spacing ratio 2.2 & \\
Uniform & 0.638 & 0.079 \\
Non-uniform & 0.590 & 0.075 \\
\hline
\end{tabular}

\subsection{Pre-processing Stage}

In the pre-processing stage, the domain's geometry in 2D was sketched, as shown in Figure 1 . The cylinder diameter, $\mathrm{d}$ was set as $1 \mathrm{~m}$. Meanwhile, the diameter of the perforated circular shroud, $\mathrm{D}$ was set according to the spacing ratio. The spacing gap ratio, defined as $D / d$, were $1.2,1.5,2$, and 2.2. The geometry of the domain was at $13.5 \mathrm{~m}$ and $20 \mathrm{~m}$ from the cylinder axis. The upper and lower boundaries were both located at $10 \mathrm{~m}$ from the cylinder axis. The detailed geometry of the 67 percent holes distribution for the perforated shrouded cylinder is shown in Figure 2. 


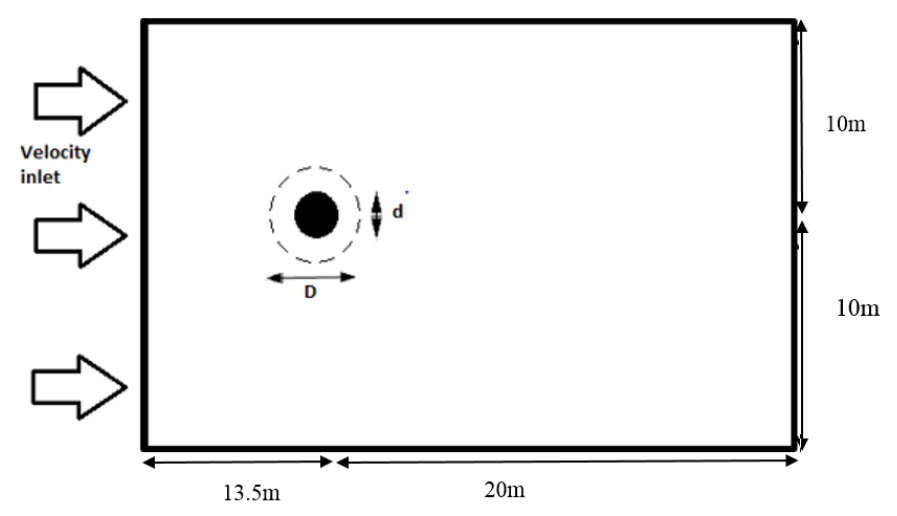

Fig. 1. The computational domain

In this study, uniform and non-uniform shrouds with a porosity of $67 \%$ were studied as the passive device to suppress the vortex shedding from a cylinder. For uniform holes distribution, the porosity, $\beta$ was calculated using Eq. (1),

$\beta=\frac{n S}{\pi D}$

where $\mathrm{n}$ is the number of holes, $S=\frac{D}{2} \theta$ is the arc length; and $\boldsymbol{\theta}=\frac{\pi}{18}$ is the hole angle set at a fixed value. By using the equation, the porosity is reduced to $\beta=\frac{n}{36}$. Based on Eq. (1), the number of holes $\mathrm{n}$ is 24 for a $67 \%$ porosity value.

For non-uniform holes, different values of theta, $\theta=5^{\circ}, 10^{\circ}, 15^{\circ}$ were used with different number of holes. The circular cylinder was divided into 2 quadrants, and $\theta=\left(\mathrm{nX} 5^{\circ}\right)+\left(n \times 10^{\circ}\right)+$ $\left(n X 15^{\circ}\right)$ in quadrant 1 . The exact angle was repeated for the second quadrant. Therefore, $\beta$ can be calculated using Eq. (2):

$\beta=\frac{\left(4 X 5^{\circ}\right)+\left(4 X 10^{\circ}\right)+\left(4 X 15^{\circ}\right)}{180^{\circ}}=0.67$

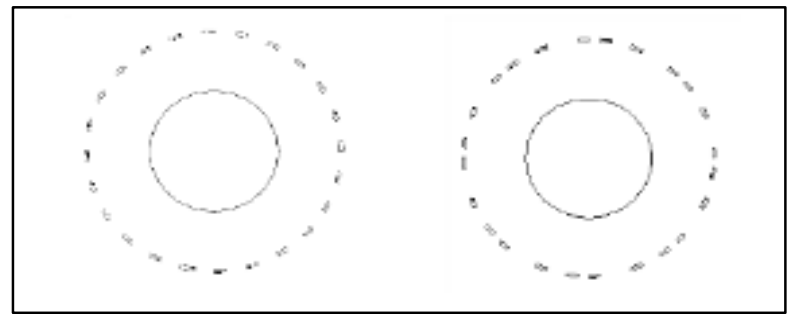

Fig. 2. Shroud geometry of $67 \%$ porosity: uniform holes (left) and non-uniform holes (right)

The next step after sketching the domain is meshing. A well-defined mesh is vital to ensure the accuracy of the results and many available methods can be used to mesh the geometry domain. The number of nodes and element of meshing is used to determine whether the mesh is coarse or fine. The near solid boundary of the cylinders must consist of fine mesh in comparison to the other areas. For this simulation, meshing was carried out utilizing several methods: refinement, edge sizing, and all triangle methods. Edge sizing was used for the circular cylinder and perforated shroud edges. Figure 3 shows the computational mesh used in this simulation. 


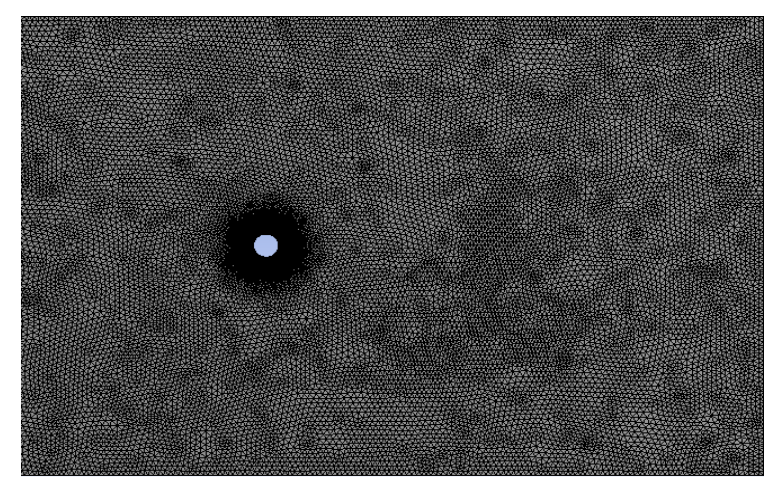

Fig. 3. Mesh structures around the perforated shrouded cylinder

\subsection{Computational Stage}

All the input parameters for the simulation were inserted into the setup for calculation during the computational stage. The solver utilizes a pressure-based type with transient time. Since the fluid flow is at $\mathrm{Re}=200$ (based on the inner cylinder diameter), the viscous laminar model was employed in the setup. In the boundary condition, the cylinder setup was in no-slip condition and stationary wall boundary conditions. The zone wall setup in specific shear condition ensures the top and bottom walls fluid will slip freely. The inlet zone type was velocity-inlet. For the solution method, Second Order Implicit was used as a transient formulation with a scheme set as Couple for better accuracy. The drag and lift coefficient setup were created in the monitor setting to obtain their values and graphs. The drag and lift coefficients were calculated from the forces acting on the cylinder, assuming that the cylinder is rigid. The automatic hybrid initialization was chosen for this simulation solution. The calculation was run at a time step size of 0.001 s for 1000 time-steps with 50 maximum iterations per time step.

\subsubsection{Grid independency study}

The grid independency study was performed to ensure the numerical accuracy of computational results and minimize the discretization error. The study was conducted by increasing the numbers of cells and nodes of the mesh in the geometry domain. The level of refinement meshing gives the difference to the results. The meshing parameter used for coarse mesh is 0.5 for default element size, 0.02 for element size of circular cylinder edge sizing, and 0.05 for element size of perforated shroud edge sizing. For medium mesh, it is 0.4 for default element size, 0.02 for element size of circular cylinder edge sizing, and 0.05 for element size of perforated shroud edge sizing.

On the other hand, fine mesh consists of 0.4 for default element size, 0.01 for element size of circular cylinder edge sizing, and 0.04 for element size of perforated shroud edge sizing. Table 2 shows the result of the grid independency study for this simulation. Further meshing refinement shows that the results from fine mesh are desirable. Thus, fine mesh is sufficient and was used for this simulation. 
Table 2

Grid independent study

\begin{tabular}{lllllll}
\hline $\begin{array}{l}\text { Spacing } \\
\text { gap ratio }\end{array}$ & $\begin{array}{l}\text { Uniform } \\
\text { Nodes }\end{array}$ & $\begin{array}{l}\text { Drag } \\
\text { Coefficient, Cd }\end{array}$ & $\begin{array}{l}\text { Strouhal } \\
\text { Number, St }\end{array}$ & $\begin{array}{l}\text { Non-uniform } \\
\text { Nodes }\end{array}$ & $\begin{array}{l}\text { Drag } \\
\text { Coefficient, Cd }\end{array}$ & $\begin{array}{l}\text { Strouhal } \\
\text { Number, St }\end{array}$ \\
\hline 1.2 & 16354 & 1.413 & 0.165 & 16329 & 1.477 & 0.165 \\
& 22872 & 1.456 & 0.165 & 22751 & 1.454 & 0.165 \\
& 27777 & 1.461 & 0.165 & 27373 & 1.472 & 0.165 \\
1.5 & 17518 & 0.994 & 0.130 & 17682 & 0.970 & 0.130 \\
& 24152 & 1.002 & 0.135 & 24072 & 0.974 & 0.135 \\
2 & 29740 & 1.008 & 0.135 & 29329 & 0.978 & 0.135 \\
& 19994 & 0.670 & 0.089 & 19926 & 0.627 & 0.075 \\
& 26120 & 0.665 & 0.085 & 26368 & 0.628 & 0.075 \\
& 31709 & 0.665 & 0.089 & 31969 & 0.627 & 0.089 \\
& 20606 & 0.645 & 0.085 & 20442 & 0.589 & 0.085 \\
& 26776 & 0.639 & 0.075 & 26612 & 0.588 & 0.085 \\
\end{tabular}

\subsection{Post-processing Stage}

In the post-processing stage, the data obtained is in the form of contour. The contours plotted are streamline, pressure coefficient, vector, velocity, and vorticity contour plot. These contours are compared with the results obtained from the computational stage.

\section{Results}

\subsection{Strouhal Number}

Figure 4 shows the power spectral density against the Strouhal number to investigate the periodicity of the flow. It shows the different magnitude of the peak, which corresponds to the vortex intensity behind the cylinder. The Strouhal number relates to the dominant shedding frequency obtained from the highest peak of power spectral density. It can be observed from Figure 4 that the Strouhal number can be further reduced by adding a shroud cylinder of a bigger spacing ratio, i.e. the vortex shedding is suppressed more efficiently with a bigger spacing ratio. The figure also indicates that the type of holes, either uniform or non-uniform, does not affecting the vortex intensity for spacing ratio of 1.5 but is significant when the ratio is greater than 2.0, evident by the slightly broader peak and lower magnitude (of the order of one-tenth) for the latter cases. Interestingly, the gap ratio of 1.2 shows higher magnitude than that of a circular cylinder.

Figure 5 shows that the Strouhal number was considerably reduced with the addition of the shrouds. Strouhal number decreases with increasing spacing ratio. However, there is not much difference in the value of the Strouhal between uniform and non-uniform hole types for all spacing ratios cases. 

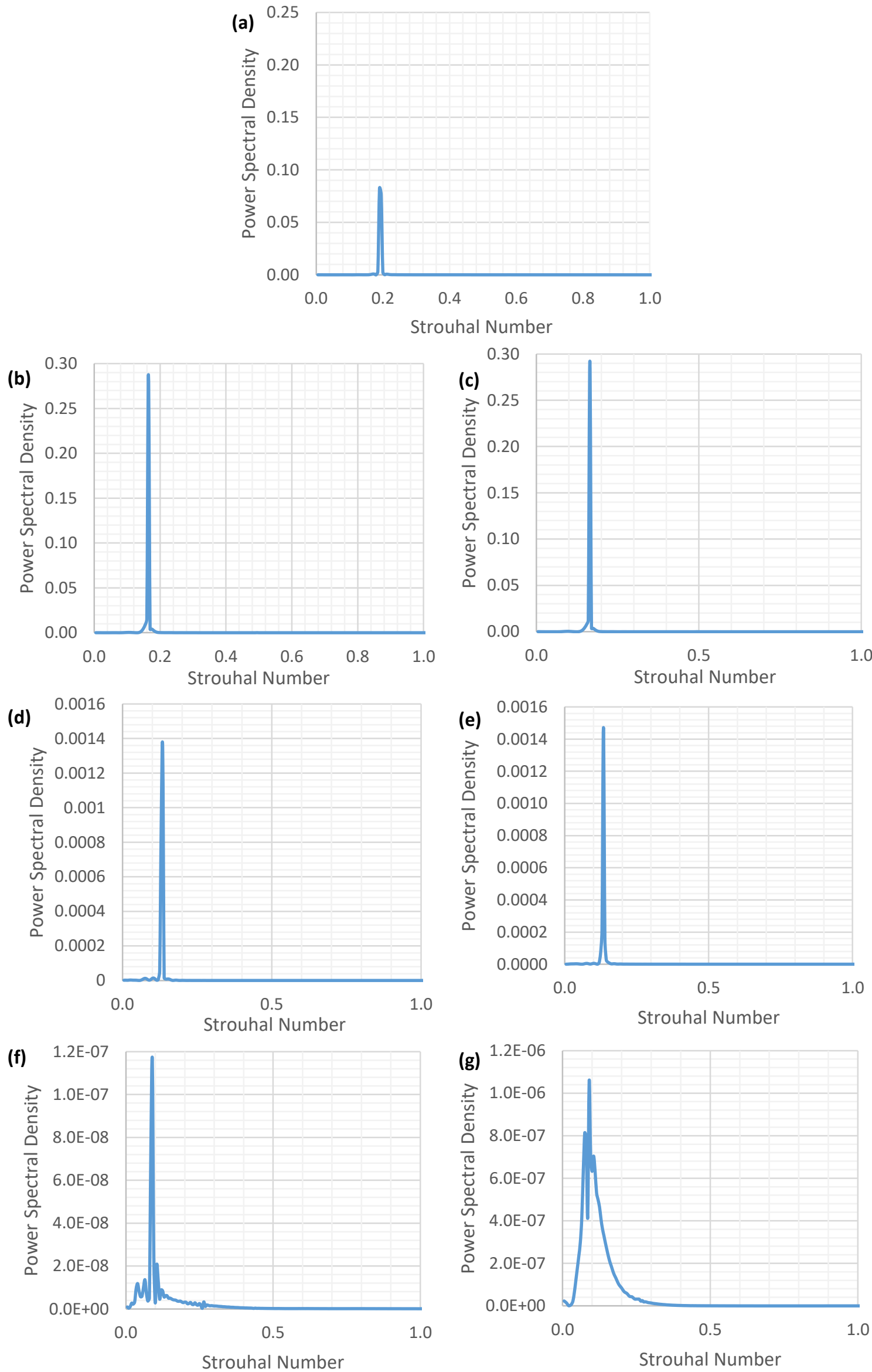

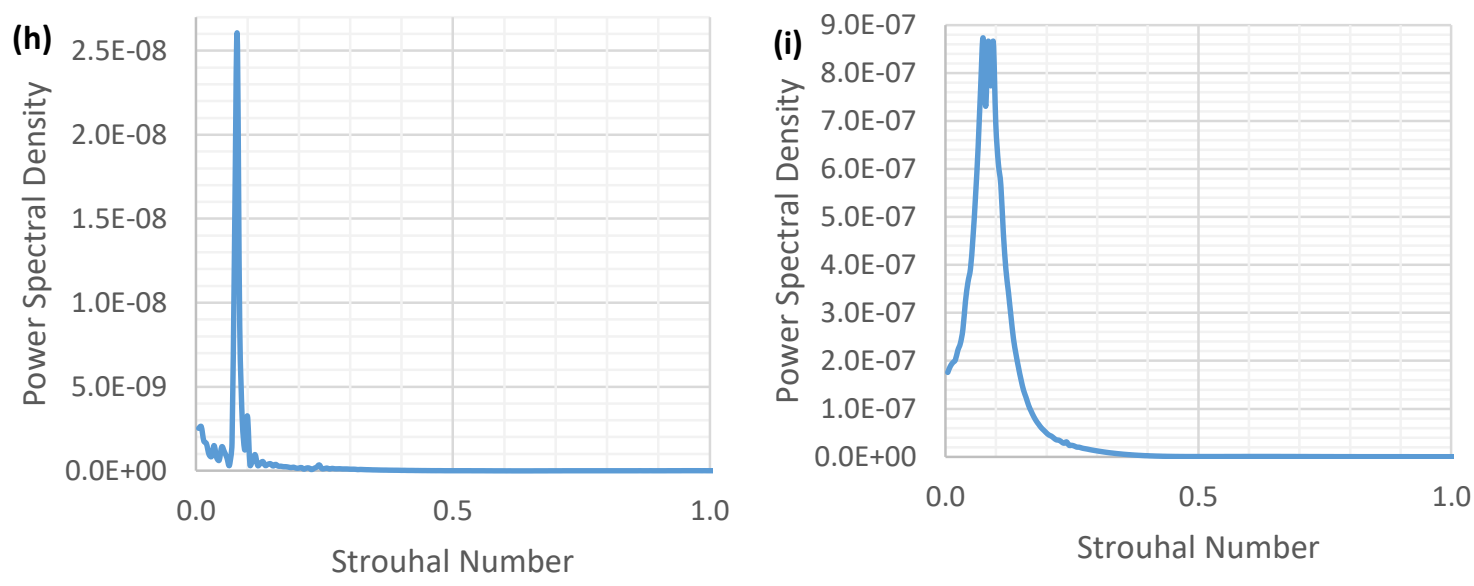

Fig. 4. Power Spectral Density vs Strouhal Number for (a) Circular Cylinder; (b) Shrouded Cylinder, gap ratio 1.2, uniform holes; (c) Shrouded Cylinder, gap ratio 1.2, non-uniform holes; (d) Shrouded Cylinder, gap ratio 1.5, uniform holes; (e) Shrouded Cylinder, gap ratio 1.5, non-uniform holes; (f) Shrouded Cylinder, gap ratio 2.0, uniform holes; (g) Shrouded Cylinder, gap ratio 2.0, non-uniform holes; (h) Shrouded Cylinder, gap ratio 2.2, uniform-holes; and (i) Shrouded Cylinder, gap ratio 2.2, non-uniform holes

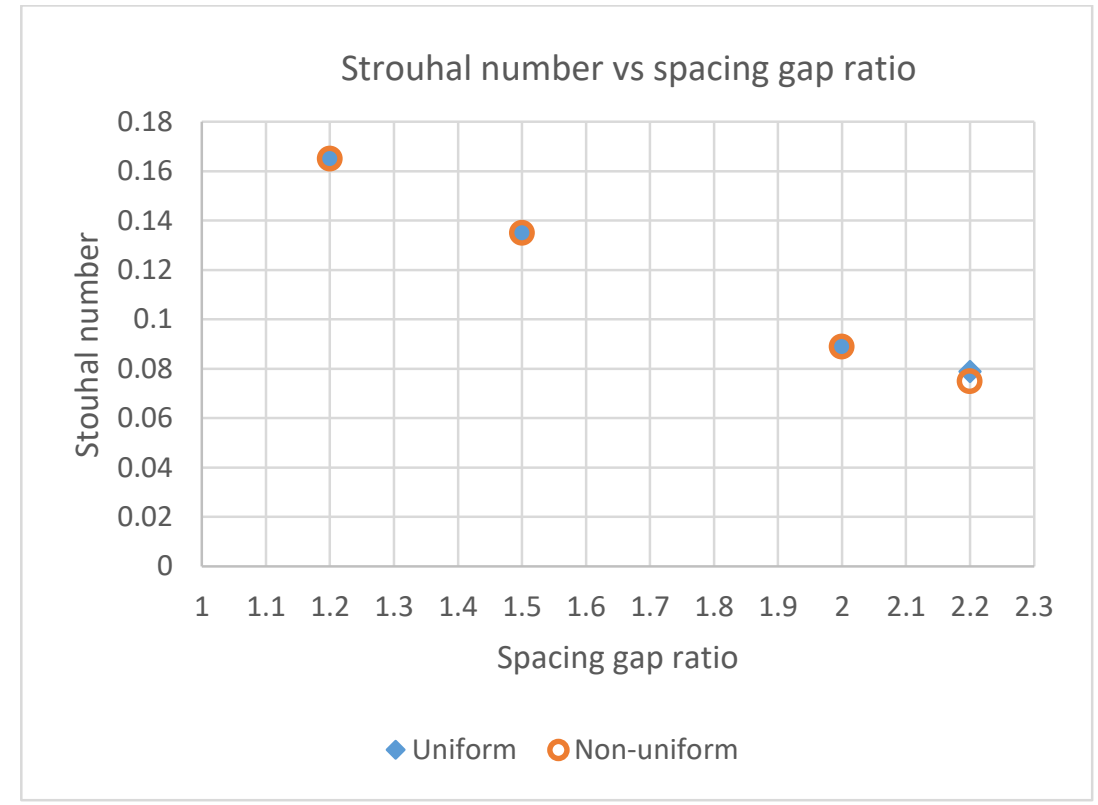

Fig. 5. Strouhal Number at various Spacing Gap Ratios

\subsection{Drag Coefficient}

Figure 6 shows the time history of the drag for all cylinder cases at spacing gap ratios of 1.2, 1.5, 2, and 2.2. It was observed that the shrouded cylinders with spacing gap ratio of 1.5, 2, and 2.2 are better in reducing the drag coefficient. However, the spacing gap ratio of 1.2 has the highest drag coefficient compared with all the other cases, consistent with relatively high Power Spectral Density magnitude discussed in Figure 4. The low drag coefficient value indicates lower resistance around the cylinder. Figure 7 indicates that the hole types: uniform or non-uniform holes, greatly influence the drag coefficient of the shrouded cylinders at spacing gap ratio greater than 2.0 , where the nonuniform holes cases give lower drag than the uniform ones. 

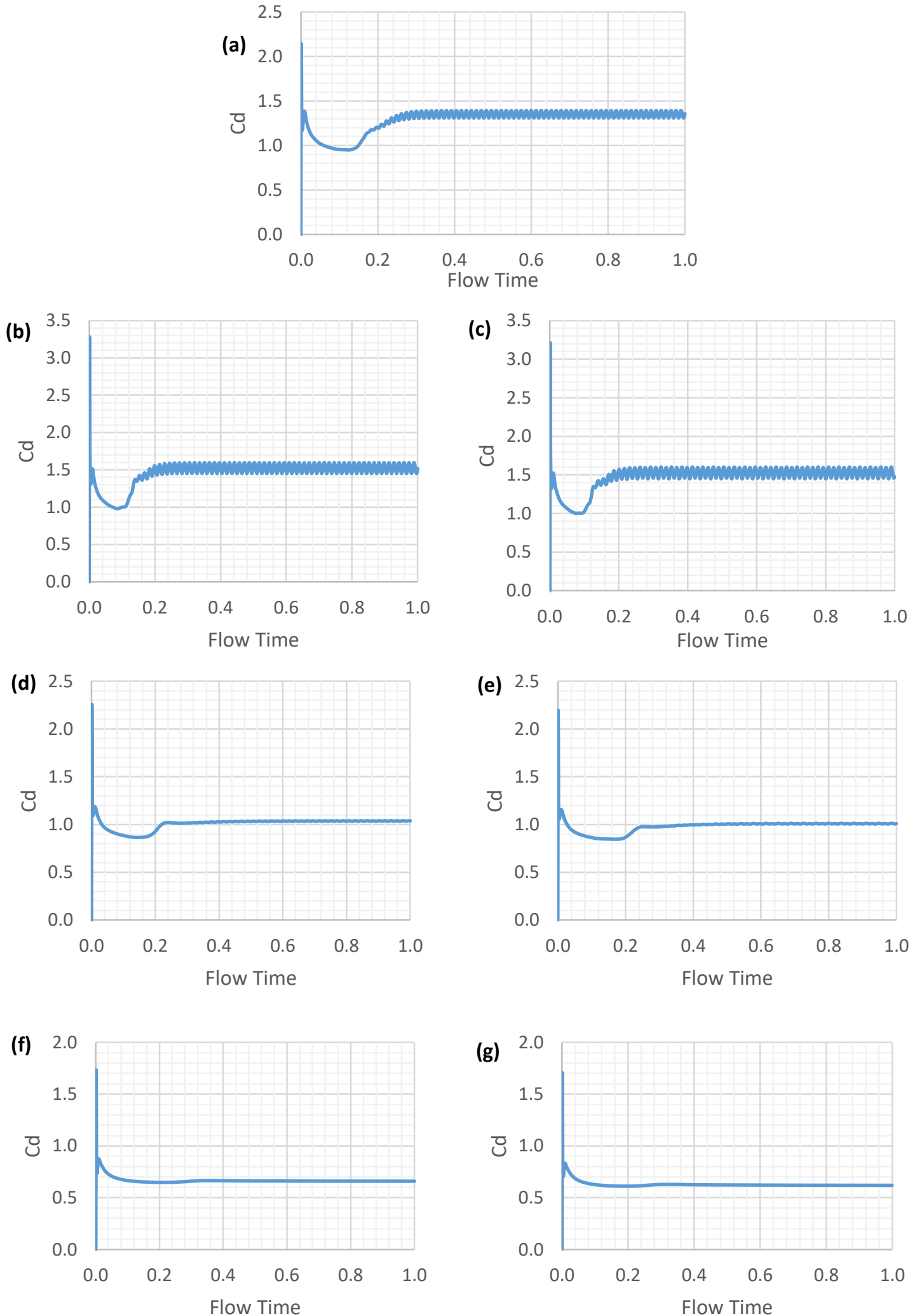

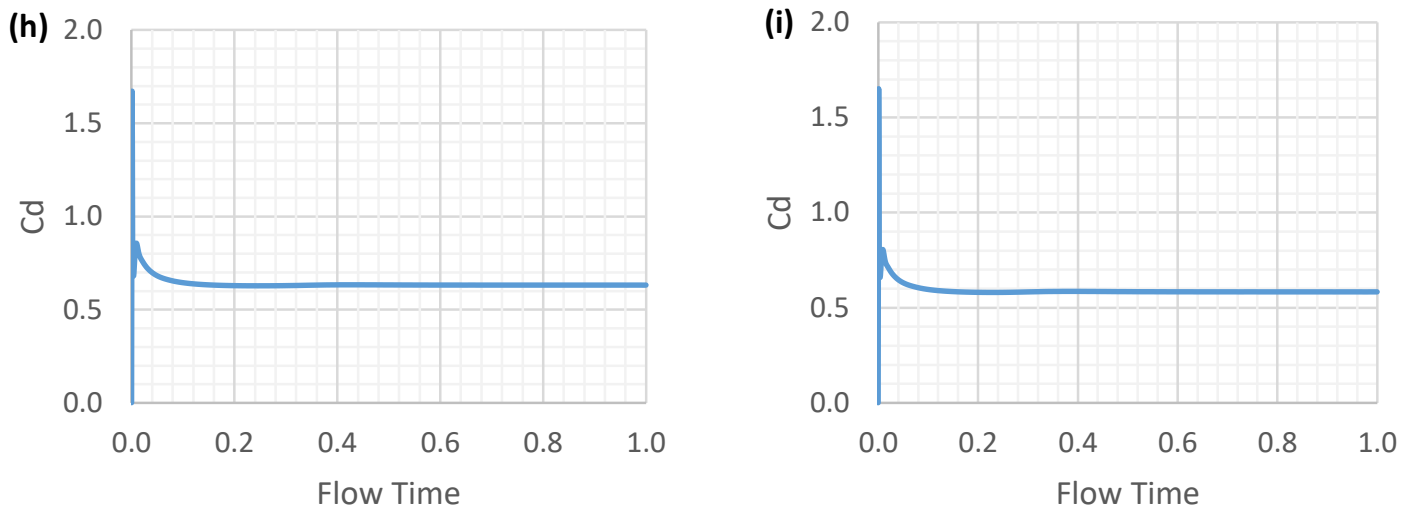

Fig. 6. Time history of Drag Coefficient for (a) Circular Cylinder; (b) Shrouded Cylinder, gap ratio 1.2, uniform holes; (c) Shrouded Cylinder, gap ratio 1.2, non-uniform holes; (d) Shrouded Cylinder, gap ratio 1.5, uniform holes; (e) Shrouded Cylinder, gap ratio 1.5, non-uniform holes; (f) Shrouded Cylinder, gap ratio 2.0, uniform holes; (g) Shrouded Cylinder, gap ratio 2.0, nonuniform holes; (h) Shrouded Cylinder, gap ratio 2.2, uniform-holes; and (i) Shrouded Cylinder, gap ratio 2.2 , non-uniform holes

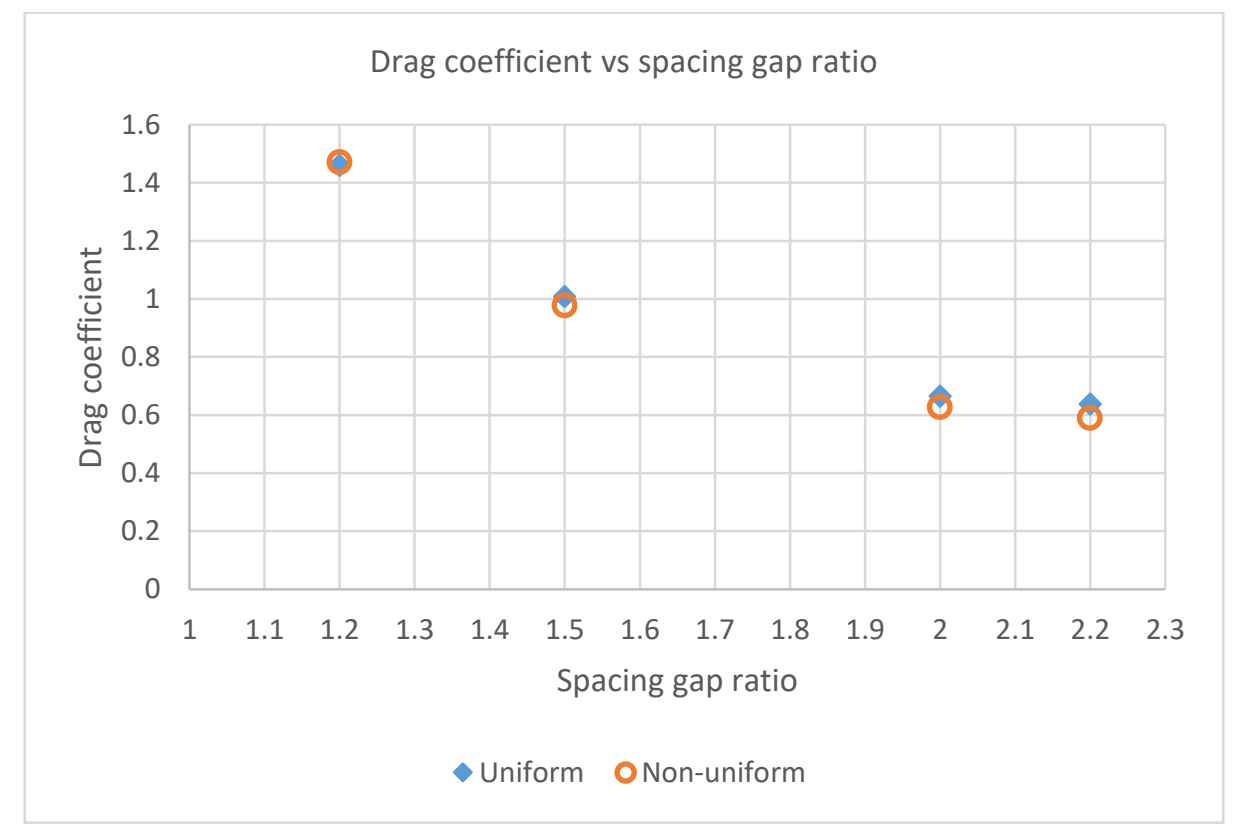

Fig. 7. Drag Coefficient at various Spacing Gap Ratio

\subsection{Lift Coefficient}

Figure 8 shows the flow time history of the lift coefficient for all cylinder wakes. Figure 8(a) shows the periodic nature of the flow in the circular cylinder due to correlated vortex shedding. It is evident that the circular cylinder has reached a steady state earlier compared to the perforated shrouded cylinders, except for the case of spacing ratio 1.2 (Figure 8(b) and Figure 8(c)). The oscillations are significantly suppressed by adding the shrouds, with greater suppression at spacing gap ratio greater than 2.0, indicating the absence of correlated vortex shedding. 

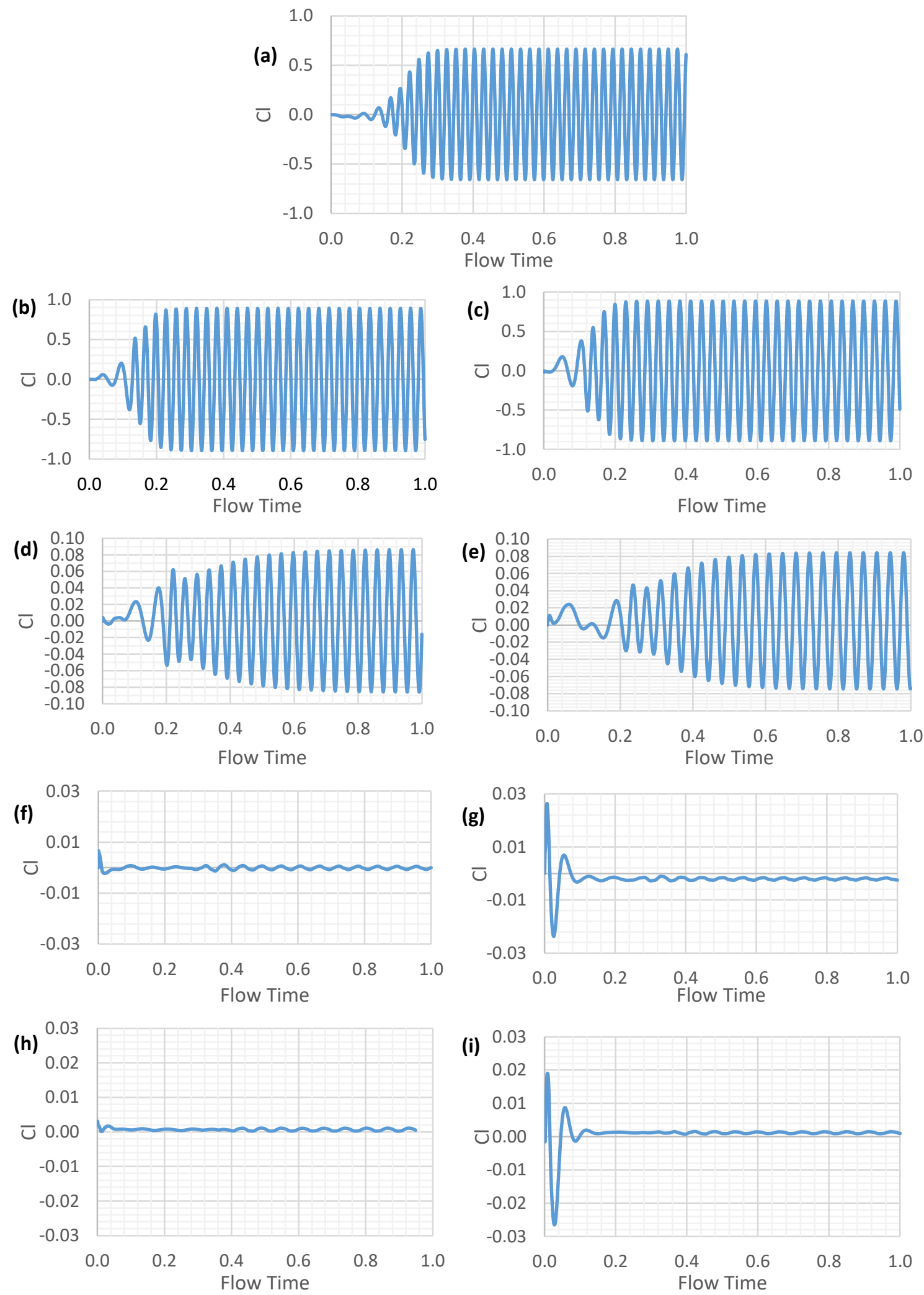

Fig. 8. Time History of Lift coefficient for (a) Circular Cylinder; (b) Shrouded Cylinder, gap ratio 1.2, uniform holes; (c) Shrouded Cylinder, gap ratio 1.2, non-uniform holes; (d) Shrouded Cylinder, gap ratio 1.5, uniform holes; (e) Shrouded Cylinder, gap ratio 1.5, non-uniform holes; (f) Shrouded Cylinder, gap ratio 2.0, uniform holes; (g) Shrouded Cylinder, gap ratio 2.0, non-uniform holes; (h) Shrouded Cylinder, gap ratio 2.2, uniform-holes; and (i) Shrouded Cylinder, gap ratio 2.2, nonuniform holes 


\subsection{Vorticity Contour}

The Von Karman vortex street is shown clearly by the vorticity contour shown in Figure 9(a). It was observed that the vortex formation length in the circular cylinder wake is much shorter compared to that of the shrouded cylinders, except for the case of spacing ratio 1.2 (Figure 9(b) and Figure 9(c)). It can be seen the length of shear layers extended farther downstream with increasing spacing gap ratio, especially for spacing ratio 2.2 (Figure 9(h) and Figure 9(i)). This is likely due to the shrouds effectively reduce the oncoming free stream's velocity towards the cylinder. This phenomenon causes the local Reynolds number to decrease and stabilizes the shear layers attached to the cylinder. There is not much difference in the formation length and vortex intensity in the wakes between the uniform and non-uniform holes shrouds.
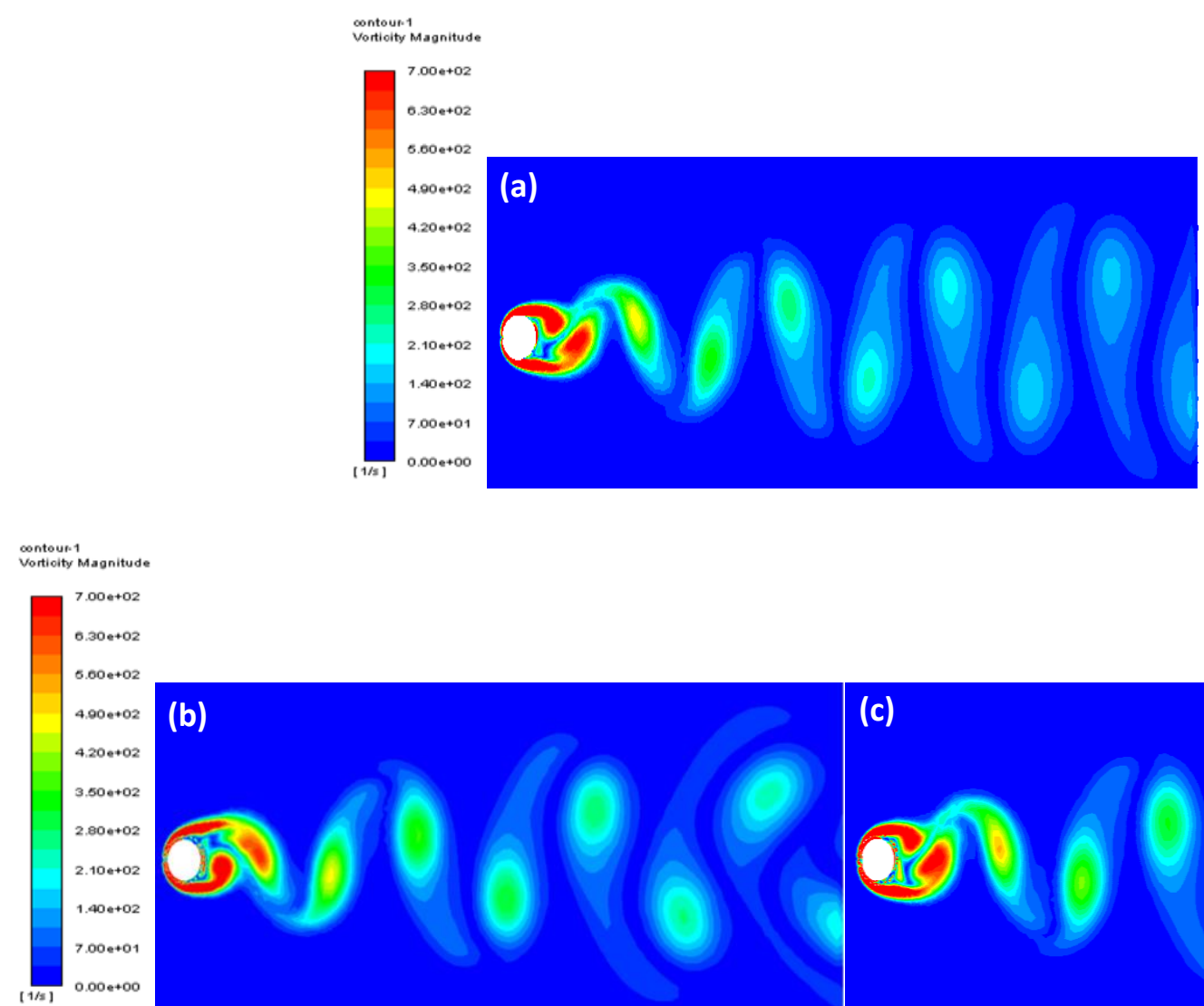

\section{(b)}

(c)

contour 1
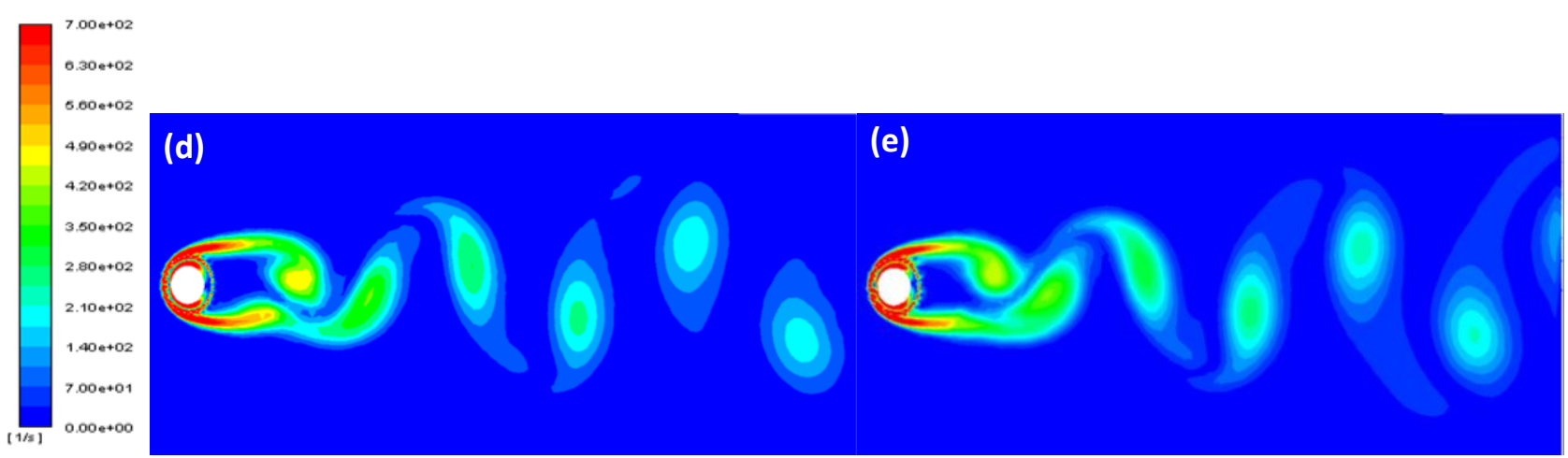

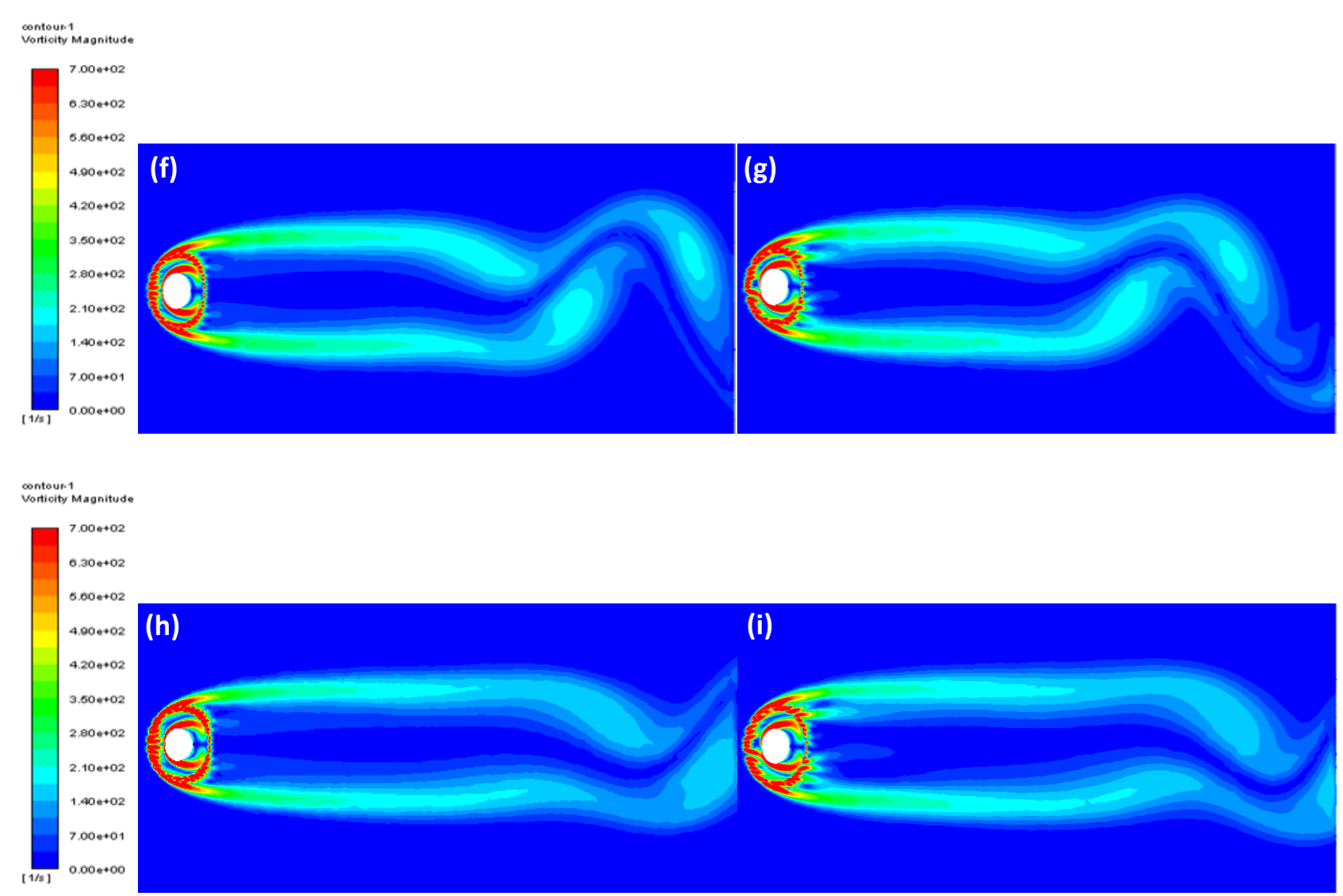

Fig. 9. Normalized Vorticity Contours (maximum value: 3.5, minimum value 0 ) in the wakes of (a) Circular Cylinder; (b) Shrouded Cylinder, gap ratio 1.2, uniform holes; (c) Shrouded Cylinder, gap ratio 1.2, nonuniform holes; (d) Shrouded Cylinder, gap ratio 1.5, uniform holes; (e) Shrouded Cylinder, gap ratio 1.5, non-uniform holes; (f) Shrouded Cylinder, gap ratio 2.0, uniform holes; (g) Shrouded Cylinder, gap ratio 2.0, non-uniform holes; (h) Shrouded Cylinder, gap ratio 2.2, uniform-holes; and (i) Shrouded Cylinder, gap ratio 2.2 , non-uniform holes

\subsection{Velocity Vector and Contour}

The velocity contours and velocity vectors in the wakes of all cylinders are shown in Figure 10 and Figure 11 respectively. The stagnation point can be seen in front of the circular cylinder and the shrouded cylinder (Figure 10(a)). The flow is greatly accelerated in the upper and lower wakes of the shrouded cylinders, particularly for the cases of spacing ratio greater than 2.0 (Figure 10(f) to Figure 10(i)). Downstream these shrouded cylinders wakes, a lengthened low-velocity region is observed which is absent in the circular cylinder wake, causing the longer vortex formation length observed in the vorticity contours discussed earlier. These shrouds effectively reduce the oncoming free stream's velocity towards the cylinder, causing the length of shear layers extended farther downstream (Figure 11(f) to Figure 11(i)). 

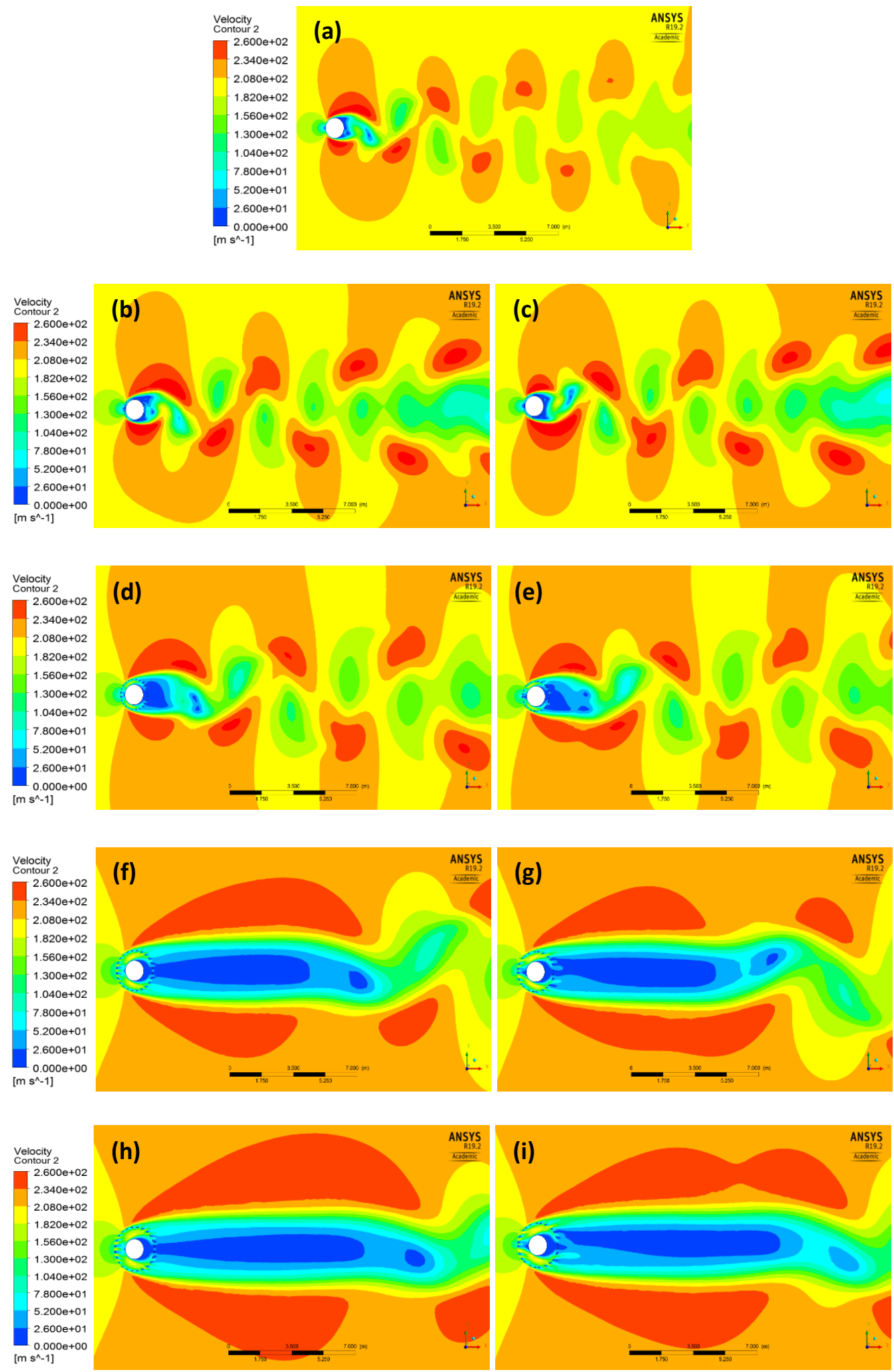

Fig. 10. Normalized Velocity Contours (maximum value: 1.3 , minimum value 0 ) in the wakes of (a) Circular Cylinder; (b) Shrouded Cylinder, gap ratio 1.2, uniform holes; (c) Shrouded Cylinder, gap ratio 1.2, non-uniform holes; (d) Shrouded Cylinder, gap ratio 1.5, uniform holes; (e) Shrouded Cylinder, gap ratio 1.5, non-uniform holes; ( $f$ ) Shrouded Cylinder, gap ratio 2.0, uniform holes; (g) Shrouded Cylinder, gap ratio 2.0, non-uniform holes; (h) Shrouded Cylinder, gap ratio 2.2, uniform-holes; and (i) Shrouded Cylinder, gap ratio 2.2, non-uniform holes 

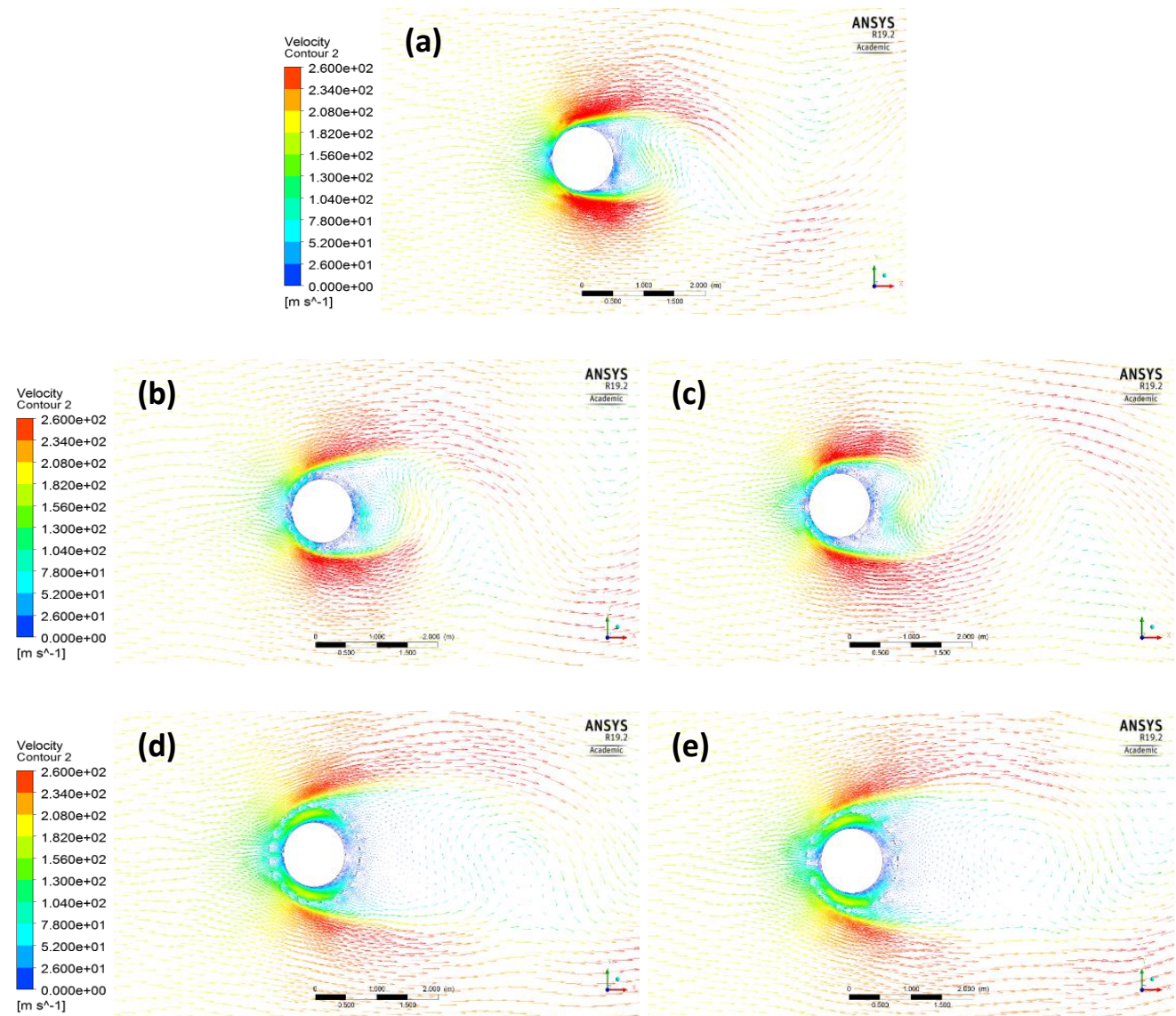

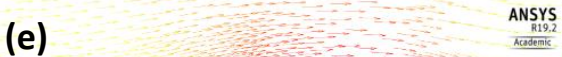

ANSYS

(g)

ANSYS

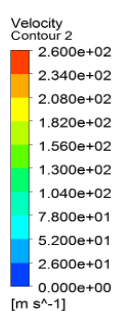

(h)

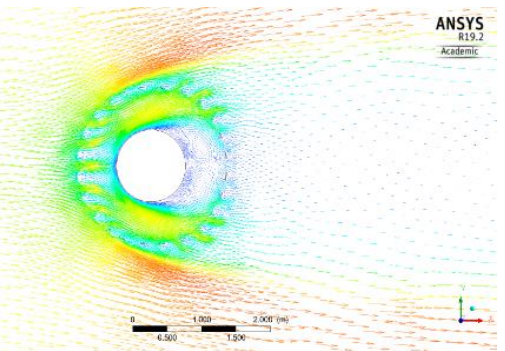

(i)
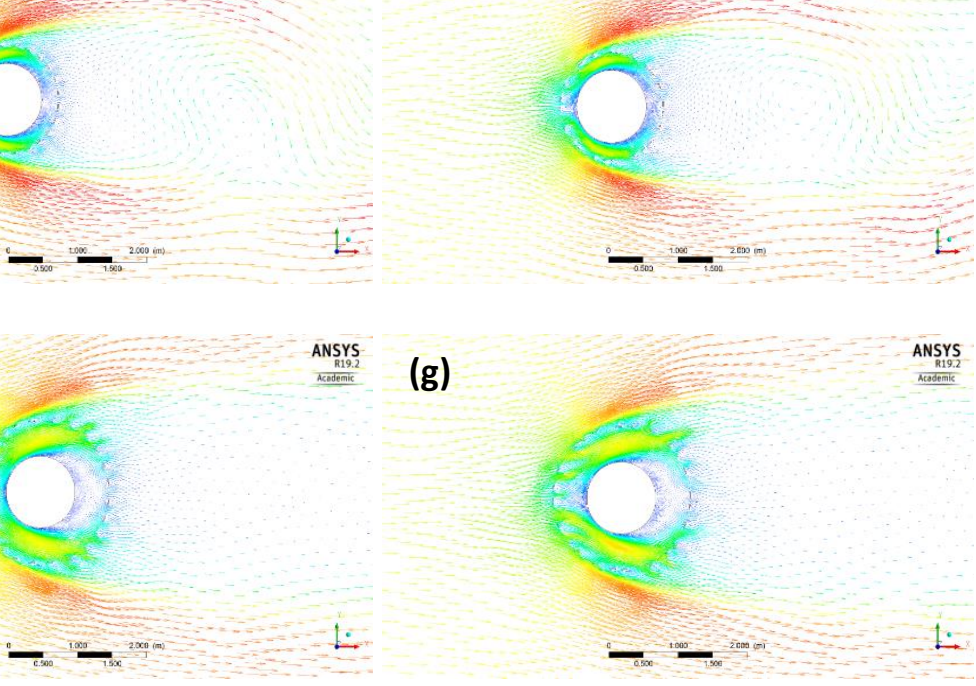

ANSYS
S19.2
acaseric.

Fig. 11. Velocity vector (maximum value: 1.3 , minimum value: 0 ) in the wakes of (a) Circular Cylinder; (b) Shrouded Cylinder, gap ratio 1.2, uniform holes; (c) Shrouded Cylinder, gap ratio 1.2, non-uniform holes; (d) Shrouded Cylinder, gap ratio 1.5, uniform holes; (e) Shrouded Cylinder, gap ratio 1.5, non-uniform holes; (f) Shrouded Cylinder, gap ratio 2.0, uniform holes; (g) Shrouded Cylinder, gap ratio 2.0, nonuniform holes; (h) Shrouded Cylinder, gap ratio 2.2, uniform-holes; and (i) Shrouded Cylinder, gap ratio 2.2, non-uniform holes 


\subsection{Pressure Contour}

Figure 12 shows the pressure contour for all cylinder wakes. The pressure contour is asymmetric for all cases. As a result of the periodic change of the vortex shedding, the cylinder's pressure distribution will change periodically due to the flow. Figure 12 clearly shows the changes in pressures from high pressure at the upstream of the cylinders to the low pressure at the downstream region of the cylinders, which give rise to drag force. This pressure difference is lessened with increasing gap ratio, particularly for spacing ratio greater than 2.0 (Figure 12(f) to Figure 12(i)), signifying attenuation of vortex resulting from relatively large annular regions.
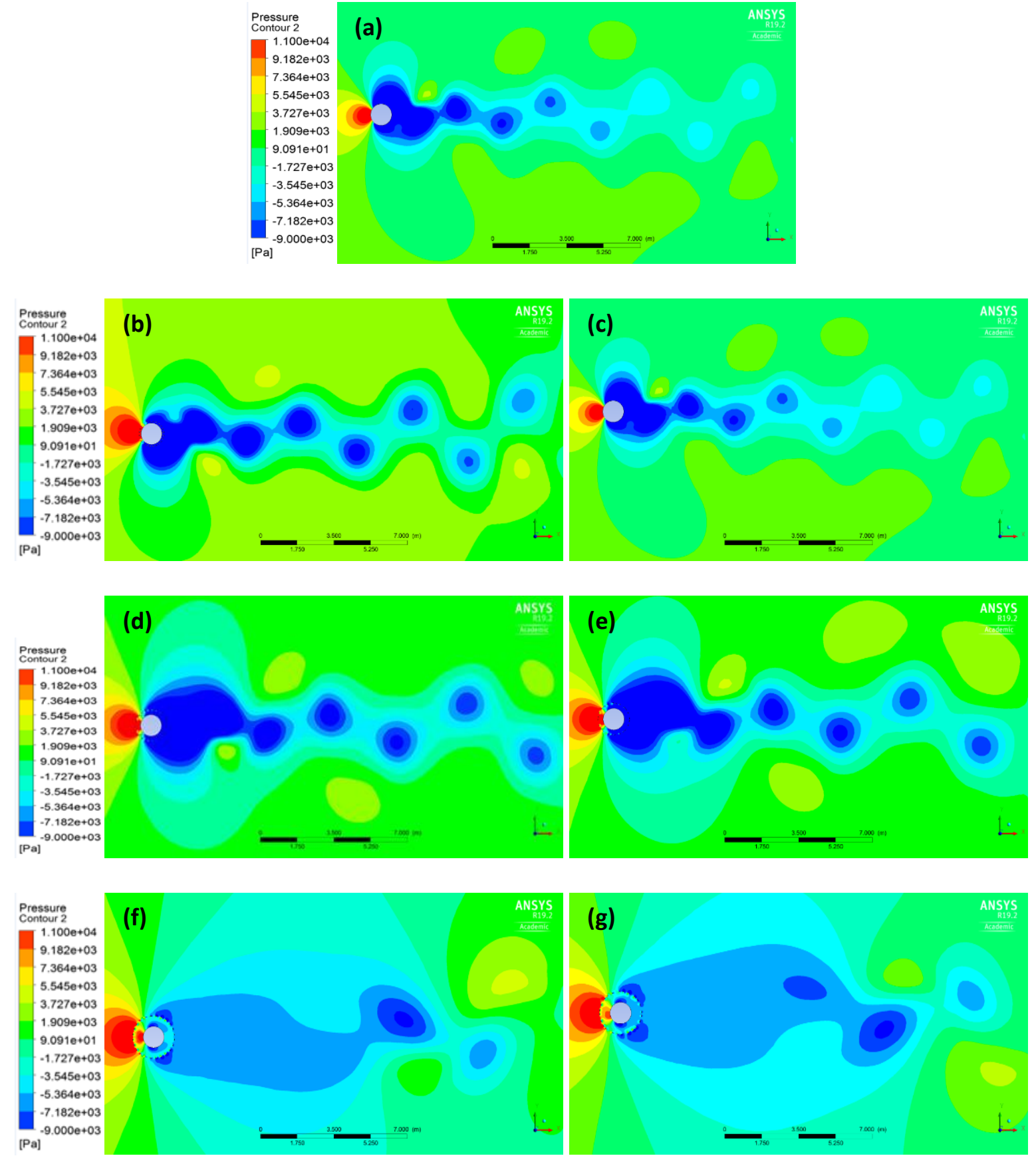


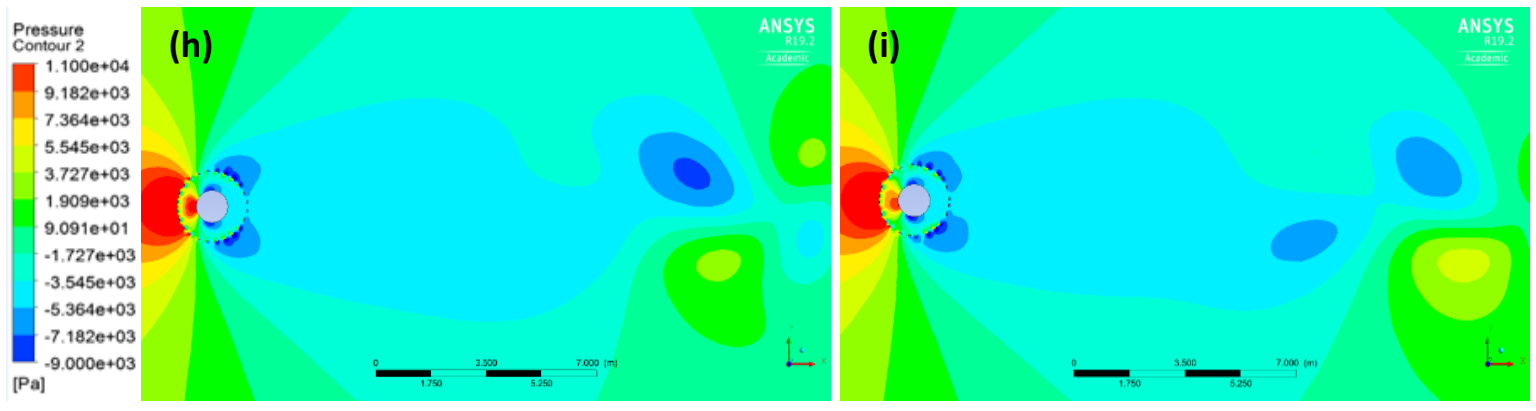

Fig. 12. Pressure contours in the wakes of (a) Circular Cylinder; (b) Shrouded Cylinder, gap ratio 1.2, uniform holes; (c) Shrouded Cylinder, gap ratio 1.2, non-uniform holes; (d) Shrouded Cylinder, gap ratio 1.5, uniform holes; (e) Shrouded Cylinder, gap ratio 1.5, non-uniform holes; (f) Shrouded Cylinder, gap ratio 2.0, uniform holes; (g) Shrouded Cylinder, gap ratio 2.0, non-uniform holes; (h) Shrouded Cylinder, gap ratio 2.2, uniform-holes; and (i) Shrouded Cylinder, gap ratio 2.2, nonuniform holes

\section{Conclusion}

CFD simulations for two-dimensional flow over a cylinder enclosed in various perforated circular shrouds at a Reynolds number of 200 were performed. The results obtained were validated with the experimental results of the existing studies in the literature. The numerical results were visualized by vorticity, velocity and pressure contours and plotted in terms of drag coefficient, lift coefficient, and Strouhal number. The effect of the shrouded cylinders and their spacing gap ratio were studied and discussed to understand the effective flow control mechanism in suppressing the vortex shedding. Results showed that the shrouded cylinders with spacing gap ratio greater than 2.0 suppress vortex significantly, signified by the longer vortex formation length, lower vortex intensity and lower value of drag coefficient in comparison to those of other cases. The effect of hole types: uniform or nonuniform, seems to be effective at spacing ratio greater than 2.0, for the Reynolds number chosen in this study.

\section{Acknowledgement}

The authors fully acknowledge Universiti Teknologi MARA for providing the 600-RMC/MyRA 5/3/LESTARI (063/2020) grant.

\section{References}

[1] Dehkordi, Behzad Ghadiri, Hesam Sarvghad Moghaddam, and Hamed Houri Jafari. "Numerical simulation of flow over two circular cylinders in tandem arrangement." Journal of Hydrodynamics 23, no. 1 (2011): 114-126. https://doi.org/10.1016/S1001-6058(10)60095-9

[2] Giosan, loan, and P. Eng. "Vortex shedding induced loads on free standing structures." Structural Vortex Shedding Response Estimation Methodology and Finite Element Simulation 42 (2013).

[3] Galbraith, R. A. McD. "Flow pattern around a shrouded cylinder at $\mathrm{Re}=5 \times 103$." Journal of Wind Engineering and Industrial Aerodynamics 6, no. 3-4 (1980): 227-242. https://doi.org/10.1016/0167-6105(80)90003-3

[4] Triantafyllou, Michael S., Rémi Bourguet, Jason Dahl, and Yahya Modarres-Sadeghi. "Vortex-induced vibrations." In Springer Handbook of Ocean Engineering, pp. 819-850. Springer, Cham, 2016. https://doi.org/10.1007/978-3-31916649-0 36

[5] Cottet, Georges-Henri, Roland Hildebrand, Petros Koumoutsakos, Chloé Mimeau, Iraj Mortazavi, and Philippe Poncet. "Passive and active flow control using vortex methods." In 6th International Conference on Vortex Flows and Vortex Models. 2014.

[6] Every, M. J., R. King, and D. S. Weaver. "Vortex-excited vibrations of cylinders and cables and their suppression." Ocean Engineering 9, no. 2 (1982): 135-157. https://doi.org/10.1016/0029-8018(82)90010-5

[7] Boorsma, K., Xin Zhang, N. Molin, and L. C. Chow. "Bluff body noise control using perforated fairings." AIAA Journal 47, no. 1 (2009): 33-43. https://doi.org/10.2514/1.32766 
[8] Ikeda, Mitsuru, and Takehisa Takaishi. "Perforated pantograph horn aeolian tone suppression mechanism." Quarterly Report of RTRI 45, no. 3 (2004): 169-174. https://doi.org/10.2219/rtriar.45.169

[9] Kleissl, Kenneth, and C. T. Georgakis. "Aerodynamic control of bridge cables through shape modification: A preliminary study." Journal of Fluids and Structures 27, no. 7 (2011): 1006-1020. https://doi.org/10.1016/i.jfluidstructs.2011.04.012

[10] Osrin, M. F., A. M. Azmi, H. Yusoff, and N. A. Razak. "Effect of shroud hole on the force characteristics of a circular cylinder." International Journal of Engineering and Advanced Technology 9, no. 1 (2019): 5929-5935. https://doi.org/10.35940/ijeat.A3030.109119

[11] Misman, M. R. A., A. M. Azmi, Z. A. Kamarulbaharin, and A. H. A. Hamid. "The Effect of Slat Opening on Vortex Shedding Behind a Circular Cylinder." International Journal of Recent Technology and Engineering (IJRTE) 8, no. 4 (2019): 6879-6885. https://doi.org/10.35940/ijrte.D5210.118419

[12] Yao, Jianfeng, Wenjuan Lou, Guohui Shen, Yong Guo, and Yuelong Xing. "Influence of inflow turbulence on the flow characteristics around a circular cylinder." Applied Sciences 9, no. 17 (2019): 3595. https://doi.org/10.3390/app9173595

[13] Sudalaimuthu, Vignesh K., and Xiaofeng Liu. "An Experimental Investigation of the Effect of Surface Perforation on Unsteady Aerodynamic Force Reduction for a Hollow Cylinder." In AIAA Scitech 2019 Forum, p. 1137. 2019. https://doi.org/10.2514/6.2019-1137

[14] Lin, Li-ming, Xing-fu Zhong, and Ying-xiang Wu. "Effect of peak perforation on flow past a conic cylinder at Re=100: drag, lift and Strouhal number." China Ocean Engineering 31, no. 3 (2017): 330-340. https://doi.org/10.1007/s13344-017-0039-9

[15] Azmi, Azlin Mohd, and Tongming Zhou. "Effect of screen geometry on the vortex formation behind a circular cylinder." Journal of Advanced Research in Fluid Mechanics and Thermal Sciences 44, no. 1 (2018): 47-54.

[16] Steiros, K., K. Kokmanian, N. Bempedelis, and M. Hultmark. "The effect of porosity on the drag of cylinders." Journal of Fluid Mechanics 901 (2020): R2. https://doi.org/10.1017/jfm.2020.606

[17] Meneghini, J. R., F. Saltara, C. L. R. Siqueira, and J. A. Ferrari Jr. "Numerical simulation of flow interference between two circular cylinders in tandem and side-by-side arrangements." Journal of Fluids and Structures 15, no. 2 (2001): 327-350. https://doi.org/10.1006/ifls.2000.0343

[18] Ding, H., C. Shu, K. S. Yeo, and D. Xu. "Numerical simulation of flows around two circular cylinders by mesh-free least square-based finite difference methods." International Journal for Numerical Methods in Fluids 53, no. 2 (2007): 305-332. https://doi.org/10.1002/fld.1281 